\section{Crystal structure of aquadioxido- (2-\{[(2-oxidoethyl)imino]methyl\}phenol- ato- $\left.\kappa^{3} \mathrm{O}, \mathrm{N}, \mathrm{O}^{\prime}\right)$ molybdenum(VI)}

\author{
Sowmianarayanan Parimala ${ }^{\mathrm{a}}$ and Parasuraman \\ Selvam ${ }^{a, b, c *}$
}

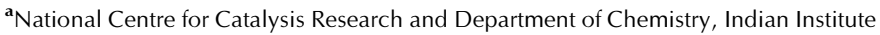
of Technology-Madras, Chennai 600 036, India, ${ }^{\mathbf{b}} \mathrm{New}$ Industry Creation Hatchery Center, Tohoku University, Sendai 980 8579, Japan, and ${ }^{\mathbf{c}}$ School of Science and Health, University of Western Australia, Sydney, Penrith, NSW 275, Australia.

*Correspondence e-mail: selvam@iitm.ac.in

Received 9 January 2015; accepted 20 January 2015

Edited by M. Weil, Vienna University of Technology, Austria

The mononuclear title complex, $\left[\mathrm{Mo}\left(\mathrm{C}_{9} \mathrm{H}_{9} \mathrm{NO}_{2}\right) \mathrm{O}_{2}\left(\mathrm{H}_{2} \mathrm{O}\right)\right]$, contains an $\mathrm{Mo}(\mathrm{VI})$ atom in a distorted octahedral coordination sphere defined by an $\mathrm{Mo}=\mathrm{O}$ and an $\mathrm{Mo}-\left(\mathrm{OH}_{2}\right)$ bond to the axial ligands and two $\mathrm{Mo}-\mathrm{O}$ bonds to phenolate and alcoholate $\mathrm{O}$ atoms, another $\mathrm{Mo}=\mathrm{O}$ bond and one $\mathrm{Mo}-\mathrm{N}$ bond to the imino $\mathrm{N}$ atom in the equatorial plane. The fivemembered metalla-ring shows an envelope conformation. In the crystal, individual molecules are connected into a layered arrangement parallel to (100) by means of $\mathrm{O}-\mathrm{H} \cdots \mathrm{O}$ hydrogen bonds involving the water molecule as a donor group and the $\mathrm{O}$ atoms of neighbouring complexes as acceptor atoms. These interactions lead to the formation of a threedimensional network.

Keywords: crystal structure; dioxidomolybdenum(VI) complex; hydrogen bonding.

CCDC reference: 1044382

\section{Related literature}

For dioxidomolybdenum complexes used as potential oxidation catalysts for the epoxidation of alkenes, see: Sakthivel et al. (2005); Masteri-Farahani et al. (2006). For chiral molybdenum complexes, see: Burke (2008); Kühn et al. (2005). These compounds are good catalysts for the oxidation of organic compounds, see: Rayati et al. (2012). For heterogenization of polymer-supported molybdenum complexes, see: Sherrington et al. (2000); Maurya (2012), and for molybdenum systems on silica supports, see: Tangestaninejad et al. (2008).

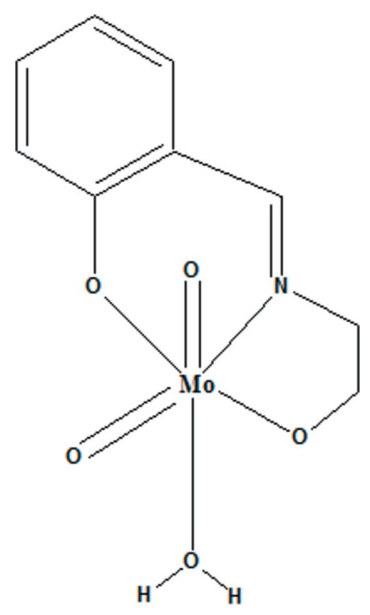

\section{Experimental}

2.1. Crystal data

$\left[\mathrm{Mo}\left(\mathrm{C}_{9} \mathrm{H}_{9} \mathrm{NO}_{2}\right) \mathrm{O}_{2}\left(\mathrm{H}_{2} \mathrm{O}\right)\right]$

$M_{r}=309.13$

Monoclinic, $P 2_{1} / c$

$a=14.9710(3) \AA$

$b=6.7026$ (1) $\AA$

$c=10.8673(2) \AA$

$\beta=99.486$ (1)

$$
\begin{aligned}
& V=1075.56(3) \AA^{3} \\
& Z=4 \\
& \text { Mo } K \alpha \text { radiation } \\
& \mu=1.22 \mathrm{~mm}^{-1} \\
& T=296 \mathrm{~K} \\
& 0.25 \times 0.16 \times 0.10 \mathrm{~mm}
\end{aligned}
$$

\subsection{Data collection}

Bruker APEXII CCD diffractometer

Absorption correction: multi-scan (SADABS; Bruker, 2012)

$T_{\min }=0.813, T_{\max }=0.934$

\subsection{Refinement}

$R\left[F^{2}>2 \sigma\left(F^{2}\right)\right]=0.016$

$w R\left(F^{2}\right)=0.042$

$S=1.10$

2392 reflections

153 parameters

Table 1

Selected bond lengths $(\AA)$.

\begin{tabular}{llll}
\hline Mo1-O5 & $1.6902(14)$ & Mo1-O2 & $1.9446(12)$ \\
Mo1-O4 & $1.7160(13)$ & Mo1-N1 & $2.2652(14)$ \\
Mo1-O1 & $1.9438(12)$ & Mo1-O3 & $2.3259(14)$ \\
\hline
\end{tabular}

Table 2

Hydrogen-bond geometry $\left(\AA{ }^{\circ}\right)$.

\begin{tabular}{lllll}
\hline$D-\mathrm{H} \cdots A$ & $D-\mathrm{H}$ & $\mathrm{H} \cdots A$ & $D \cdots A$ & $D-\mathrm{H} \cdots A$ \\
\hline $\mathrm{O} 3-\mathrm{H} 1 O \cdots \mathrm{O} 1^{\mathrm{i}}$ & $0.73(2)$ & $1.97(3)$ & $2.6656(19)$ & $161(3)$ \\
$\mathrm{O} 3-\mathrm{H} 2 O \cdots \mathrm{O} 4^{\mathrm{ii}}$ & $0.78(3)$ & $2.07(3)$ & $2.8425(19)$ & $173(3)$ \\
\hline
\end{tabular}

Symmetry codes: (i) $-x+1,-y,-z+1$; (ii) $x,-y+\frac{1}{2}, z-\frac{1}{2}$.
8837 measured reflections 2392 independent reflections 2263 reflections with $I>2 \sigma(I)$ $R_{\text {int }}=0.012$ independent and constrained refinement

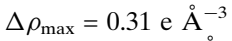

$\Delta \rho_{\min }=-0.30 \mathrm{e}^{-3}$ 
Data collection: APEX2 (Bruker, 2012); cell refinement: SAINT (Bruker, 2012); data reduction: SAINT; program(s) used to solve structure: SHELXS97 (Sheldrick, 2008); program(s) used to refine structure: SHELXL97 (Sheldrick, 2015); molecular graphics: ORTEP-3 for Windows (Farrugia, 2012); software used to prepare material for publication: publCIF (Westrip, 2010).

\section{Acknowledgements}

The authors thank the Department of Science and Technology (DST), Government of India, for funding the National Centre for Catalysis Research (NCCR), IIT-Madras. They also thank Mr V. Ramkumar and Dr R. Jagan for the data collection and technical assistance in the preparation of the manuscript.

Supporting information for this paper is available from the IUCr electronic archives (Reference: WM5114).

\section{References}

Bruker (2012). APEX2, SAINT and SADABS. Bruker AXS Inc., Madison, Wisconsin, USA.

Burke, A. (2008). Coord. Chem. Rev. 252, 170-175.

Farrugia, L. J. (2012). J. Appl. Cryst. 45, 849-854.

Kühn, F. E. J., Zhao, J. \& Herrmann, W. A. (2005). Tetrahedron Asymmetry, 16, 3469-3479.

Masteri-Farahani, M., Farzaneh, F. \& Ghandi, M. J. (2006). J. Mol. Catal. A Chem. 248, 53-60.

Maurya, R. (2012). Curr. Org Chem. 16, 73-88.

Rayati, S., Rafiee, N. \& Wojtczak, A. (2012). Inorg. Chim. Acta, 386, 27-35.

Sakthivel, A., Zhao, J., Raudaschl-Sieber, G., Hanzlik, M., Chiang, A. S. T. \& Kühn, F. E. (2005). Appl. Catal. Gen. 281, 267-273.

Sheldrick, G. M. (2008). Acta Cryst. A64, 112-122.

Sheldrick, G. M. (2015). Acta Cryst. C71, 3-8.

Sherrington, D. C. (2000). Catal. Today, 57, 87-104.

Tangestaninejad, S., Moghadam, M., Mirkhani, V., Mohammadpoor-Baltork, I. \& Ghani, K. (2008). J. Iran Chem. Soc. 5, s71-S79.

Westrip, S. P. (2010). J. Appl. Cryst. 43, 920-925. 


\section{supporting information}

Acta Cryst. (2015). E71, m35-m36 [doi:10.1107/S2056989015001231]

\section{Crystal structure of aquadioxido(2-\{[(2-oxidoethyl)imino]methyl\}phenolato- $\left.\kappa^{3} O, N, O^{\prime}\right)$ molybdenum(VI)}

\section{Sowmianarayanan Parimala and Parasuraman Selvam}

\section{S1. Experimental}

Molybdenyl acetylacetone $\left(\mathrm{MoO}_{2}(\mathrm{acac})_{2}\right)(4.03 \mathrm{~g}, 0.012 \mathrm{~mol})$ dissolved in methanol $(20 \mathrm{ml})$ was added to a refluxing solution of salicylaldehyde $(2.62 \mathrm{ml}, 0.012 \mathrm{~mol})$ and ethanolamine $(1.5 \mathrm{ml}, 0.012 \mathrm{~mol})$ in ethanol $(30 \mathrm{ml})$. The mixture was refluxed for five hours, and the solvent removed under vacuum at room temperature. The resulting yellow solution was filtered, evaporated slowly, to yield yellow crystals. The crystals were purified by washing with ethanol/methanol mixture and dried at room temperature. The obtained crystals have incorporated water. The used solvents ethanol and methanol have not been dried prior to the reaction and thus contain water. Another source of water is the condensation reaction between salicylaldehyde and ethanolamine.

\section{S2. Refinement}

All $\mathrm{H}$ atoms were identified from difference electron density maps. However, $\mathrm{C}$-bound $\mathrm{H}$ atoms were treated as riding with $\mathrm{C}-\mathrm{H}=0.97 \AA$ for $\left(\mathrm{CH}_{2}\right)$ and $\mathrm{C}-\mathrm{H}=0.93 \AA$ for aromatic $\mathrm{H}$ atoms, both with $U_{\text {iso }}(\mathrm{H})=1.2 U_{\text {eq. }}$. The $\mathrm{H}$ atoms of the water molecule were refined freely.

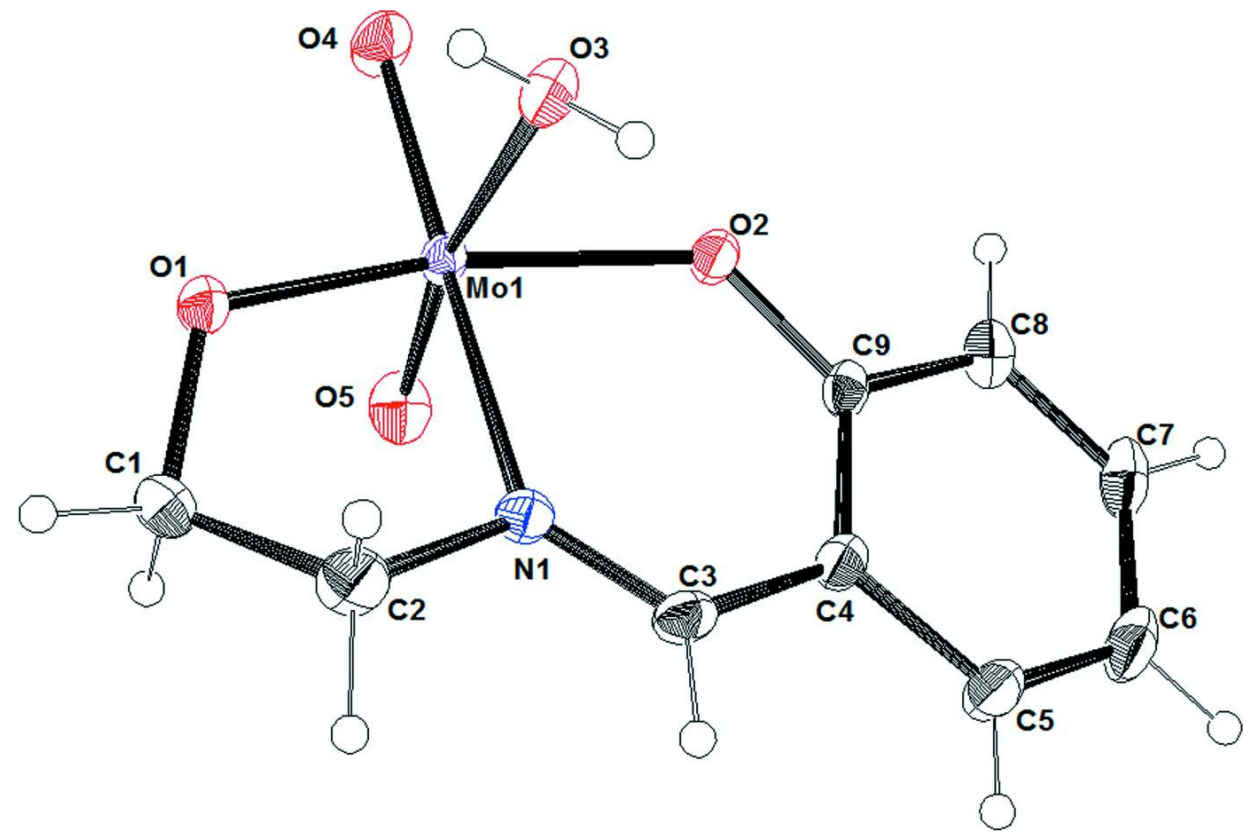

\section{Figure 1}

The molecular structure of the title compound with displacement ellipsoids drawn at the $30 \%$ probability level. 


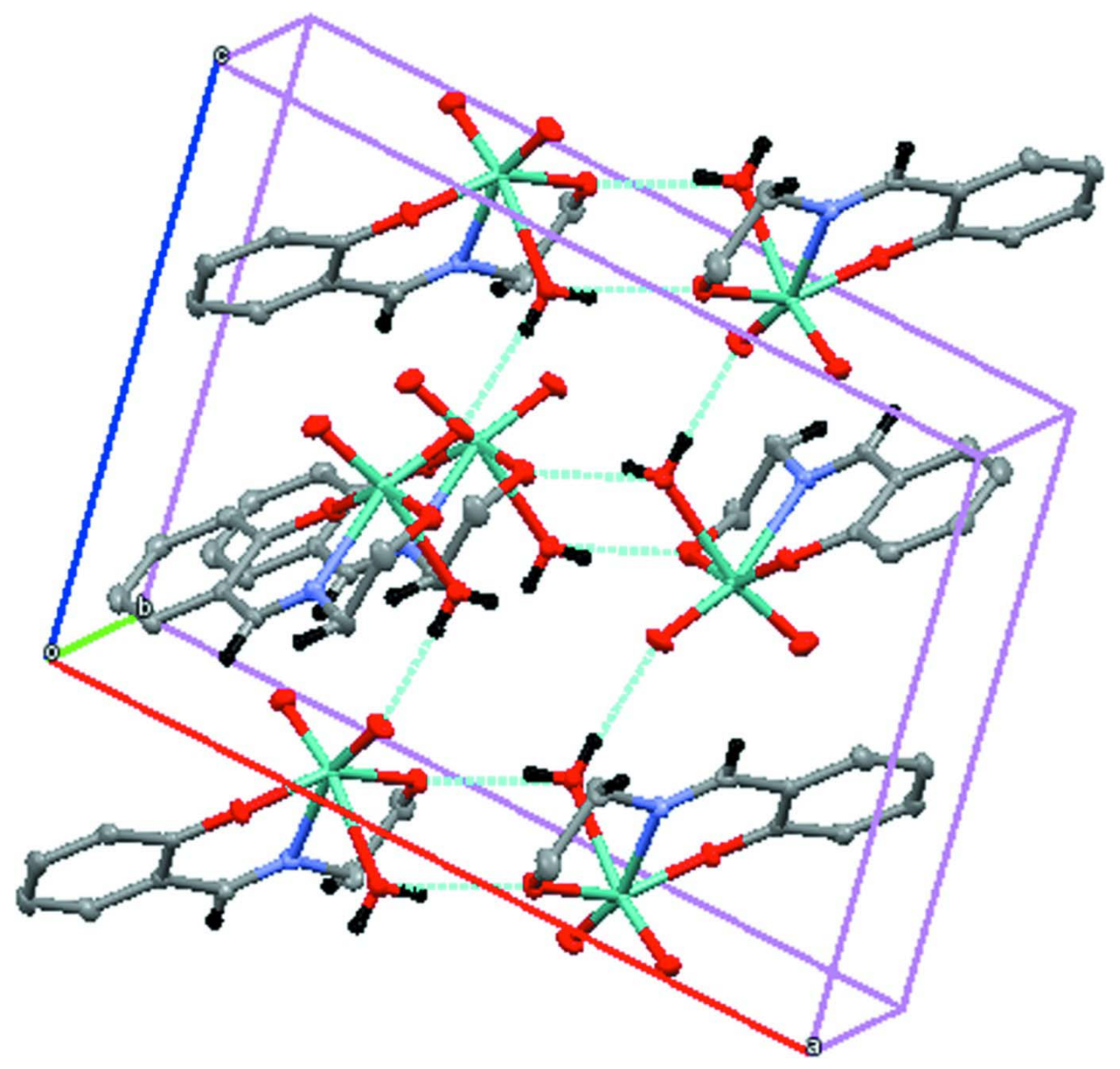

Figure 2

Unit-cell packing diagram of the title compound with hydrogen bonds shown as dashed lines. Hydrogen atoms not involved in hydrogen bonding are omitted for clarity.

\section{Aquadioxido(2-\{[(2-oxidoethyl)imino]methyl\}phenolato- $\left.\kappa^{3} O, N, O^{\prime}\right)$ molybdenum(VI)}

Crystal data

$\left[\mathrm{Mo}\left(\mathrm{C}_{9} \mathrm{H}_{9} \mathrm{NO}_{2}\right) \mathrm{O}_{2}\left(\mathrm{H}_{2} \mathrm{O}\right)\right]$

$M_{r}=309.13$

Monoclinic, $P 2{ }_{1} / c$

$a=14.9710(3) \AA$

$b=6.7026(1) \AA$

$c=10.8673(2) \AA$

$\beta=99.486(1)^{\circ}$

$V=1075.56(3) \AA^{3}$

$Z=4$

$F(000)=616$

$D_{\mathrm{x}}=1.909 \mathrm{Mg} \mathrm{m}^{-3}$

Mo $K \alpha$ radiation, $\lambda=0.71073 \AA$

Cell parameters from 6907 reflections

$\theta=2.8-27.2^{\circ}$

$\mu=1.22 \mathrm{~mm}^{-1}$

$T=296 \mathrm{~K}$

Block, yellow

$0.25 \times 0.16 \times 0.10 \mathrm{~mm}$

Data collection

Bruker APEXII CCD

diffractometer

$\varphi$ and $\omega$ scans

Absorption correction: multi-scan

(SADABS; Bruker, 2012)

$T_{\min }=0.813, T_{\max }=0.934$

8837 measured reflections

2392 independent reflections

2263 reflections with $I>2 \sigma(I)$

$R_{\text {int }}=0.012$ 
$\theta_{\text {max }}=27.2^{\circ}, \theta_{\text {min }}=1.4^{\circ}$

$h=-19 \rightarrow 18$

Refinement

Refinement on $F^{2}$

Least-squares matrix: full

$R\left[F^{2}>2 \sigma\left(F^{2}\right)\right]=0.016$

$w R\left(F^{2}\right)=0.042$

$S=1.10$

2392 reflections

153 parameters

0 restraints

$$
\begin{aligned}
& k=-8 \rightarrow 8 \\
& l=-13 \rightarrow 13
\end{aligned}
$$

Hydrogen site location: mixed

$\mathrm{H}$ atoms treated by a mixture of independent and constrained refinement

$w=1 /\left[\sigma^{2}\left(F_{\mathrm{o}}^{2}\right)+(0.0163 P)^{2}+0.8254 P\right]$ where $P=\left(F_{\mathrm{o}}^{2}+2 F_{\mathrm{c}}^{2}\right) / 3$

$(\Delta / \sigma)_{\max }=0.001$

$\Delta \rho_{\max }=0.31$ e $\AA^{-3}$

$\Delta \rho_{\min }=-0.30$ e $\AA^{-3}$

\section{Special details}

Geometry. All e.s.d.'s (except the e.s.d. in the dihedral angle between two 1.s. planes) are estimated using the full covariance matrix. The cell e.s.d.'s are taken into account individually in the estimation of e.s.d.'s in distances, angles and torsion angles; correlations between e.s.d.'s in cell parameters are only used when they are defined by crystal symmetry. An approximate (isotropic) treatment of cell e.s.d.'s is used for estimating e.s.d.'s involving 1.s. planes.

Fractional atomic coordinates and isotropic or equivalent isotropic displacement parameters $\left(\hat{A}^{2}\right)$

\begin{tabular}{lllll}
\hline & $x$ & $y$ & $z$ & $U_{\text {iso }} * / U_{\text {eq }}$ \\
\hline C1 & $0.37800(15)$ & $-0.3126(3)$ & $0.4461(2)$ & $0.0394(5)$ \\
H1A & 0.3281 & -0.3758 & 0.4778 & $0.047^{*}$ \\
H1B & 0.4281 & -0.4060 & 0.4541 & $0.047^{*}$ \\
C2 & $0.34936(14)$ & $-0.2553(3)$ & $0.31132(19)$ & $0.0384(5)$ \\
H2A & 0.4018 & -0.2223 & 0.2734 & $0.046^{*}$ \\
H2B & 0.3169 & -0.3641 & 0.2649 & $0.046^{*}$ \\
C3 & $0.22629(12)$ & $-0.0467(3)$ & $0.22157(16)$ & $0.0288(4)$ \\
H3 & 0.2174 & -0.1379 & 0.1562 & $0.035^{*}$ \\
C4 & $0.16677(11)$ & $0.1238(3)$ & $0.21392(16)$ & $0.0267(4)$ \\
C5 & $0.09354(13)$ & $0.1343(3)$ & $0.11473(18)$ & $0.0371(4)$ \\
H5 & 0.0846 & 0.0313 & 0.0567 & $0.044^{*}$ \\
C6 & $0.03507(13)$ & $0.2930(4)$ & $0.10178(19)$ & $0.0427(5)$ \\
H6 & -0.0136 & 0.2961 & 0.0365 & $0.051^{*}$ \\
C7 & $0.04887(13)$ & $0.4486(4)$ & $0.1865(2)$ & $0.0404(5)$ \\
H7 & 0.0090 & 0.5561 & 0.1780 & $0.049^{*}$ \\
C8 & $0.12118(13)$ & $0.4458(3)$ & $0.28334(18)$ & $0.0331(4)$ \\
H8 & 0.1304 & 0.5525 & 0.3387 & $0.040^{*}$ \\
C9 & $0.18051(11)$ & $0.2837(3)$ & $0.29870(15)$ & $0.0244(3)$ \\
Mo1 & $0.32157(2)$ & $0.08935(2)$ & $0.49415(2)$ & $0.02134(5)$ \\
N1 & $0.29025(10)$ & $-0.0807(2)$ & $0.31202(14)$ & $0.0254(3)$ \\
O1 & $0.40476(8)$ & $-0.13539(19)$ & $0.51476(12)$ & $0.0286(3)$ \\
O2 & $0.25056(8)$ & $0.29088(18)$ & $0.39282(11)$ & $0.0275(3)$ \\
O3 & $0.42590(10)$ & $0.2153(2)$ & $0.37755(13)$ & $0.0306(3)$ \\
O4 & $0.37757(10)$ & $0.2275(2)$ & $0.61586(12)$ & $0.0372(3)$ \\
O5 & $0.23417(9)$ & $-0.0248(2)$ & $0.54594(13)$ & $0.0392(3)$ \\
H1O & $0.4715(17)$ & $0.176(4)$ & $0.395(2)$ & $0.037(7)^{*}$ \\
H2O & $0.4144(17)$ & $0.221(4)$ & $0.305(3)$ & $0.048(7)^{*}$ \\
& & & & \\
\hline & & & &
\end{tabular}


Atomic displacement parameters $\left(\AA^{2}\right)$

\begin{tabular}{lllllll}
\hline & $U^{11}$ & $U^{22}$ & $U^{33}$ & $U^{12}$ & $U^{13}$ & $U^{23}$ \\
\hline C1 & $0.0454(11)$ & $0.0219(9)$ & $0.0463(12)$ & $0.0080(8)$ & $-0.0058(9)$ & $-0.0057(8)$ \\
C2 & $0.0417(11)$ & $0.0331(10)$ & $0.0382(11)$ & $0.0118(8)$ & $-0.0003(8)$ & $-0.0154(8)$ \\
C3 & $0.0325(9)$ & $0.0312(9)$ & $0.0219(8)$ & $-0.0039(7)$ & $0.0024(7)$ & $-0.0059(7)$ \\
C4 & $0.0239(8)$ & $0.0331(9)$ & $0.0223(8)$ & $-0.0025(7)$ & $0.0019(6)$ & $0.0033(7)$ \\
C5 & $0.0344(10)$ & $0.0460(12)$ & $0.0274(9)$ & $-0.0061(9)$ & $-0.0045(8)$ & $0.0021(9)$ \\
C6 & $0.0301(10)$ & $0.0601(14)$ & $0.0341(10)$ & $-0.0005(9)$ & $-0.0064(8)$ & $0.0154(10)$ \\
C7 & $0.0325(10)$ & $0.0508(13)$ & $0.0384(11)$ & $0.0142(9)$ & $0.0068(8)$ & $0.0183(10)$ \\
C8 & $0.0351(10)$ & $0.0363(10)$ & $0.0286(9)$ & $0.0097(8)$ & $0.0075(7)$ & $0.0061(8)$ \\
C9 & $0.0231(8)$ & $0.0295(9)$ & $0.0207(8)$ & $0.0010(7)$ & $0.0038(6)$ & $0.0059(7)$ \\
Mo1 & $0.02586(8)$ & $0.02184(8)$ & $0.01569(8)$ & $0.00375(5)$ & $0.00161(5)$ & $-0.00128(5)$ \\
N1 & $0.0283(7)$ & $0.0234(7)$ & $0.0241(7)$ & $0.0011(6)$ & $0.0031(6)$ & $-0.0047(6)$ \\
O1 & $0.0297(6)$ & $0.0241(6)$ & $0.0295(6)$ & $0.0054(5)$ & $-0.0023(5)$ & $-0.0007(5)$ \\
O2 & $0.0306(6)$ & $0.0243(6)$ & $0.0247(6)$ & $0.0054(5)$ & $-0.0035(5)$ & $-0.0023(5)$ \\
O3 & $0.0250(7)$ & $0.0404(8)$ & $0.0251(7)$ & $0.0017(6)$ & $0.0007(5)$ & $0.0052(6)$ \\
O4 & $0.0479(8)$ & $0.0381(8)$ & $0.0219(6)$ & $0.0062(6)$ & $-0.0049(6)$ & $-0.0085(6)$ \\
O5 & $0.0370(7)$ & $0.0451(8)$ & $0.0382(8)$ & $0.0037(6)$ & $0.0144(6)$ & $0.0072(7)$ \\
& & & & & & \\
\hline
\end{tabular}

Geometric parameters $\left(\AA,{ }^{\circ}\right)$

\begin{tabular}{|c|c|c|c|}
\hline $\mathrm{C} 1-\mathrm{O} 1$ & $1.425(2)$ & C6- 6 6 & 0.9300 \\
\hline $\mathrm{C} 1-\mathrm{C} 2$ & $1.507(3)$ & $\mathrm{C} 7-\mathrm{C} 8$ & $1.380(3)$ \\
\hline $\mathrm{C} 1-\mathrm{H} 1 \mathrm{~A}$ & 0.9700 & $\mathrm{C} 7-\mathrm{H} 7$ & 0.9300 \\
\hline $\mathrm{C} 1-\mathrm{H} 1 \mathrm{~B}$ & 0.9700 & $\mathrm{C} 8-\mathrm{C} 9$ & $1.396(2)$ \\
\hline $\mathrm{C} 2-\mathrm{N} 1$ & $1.468(2)$ & $\mathrm{C} 8-\mathrm{H} 8$ & 0.9300 \\
\hline $\mathrm{C} 2-\mathrm{H} 2 \mathrm{~A}$ & 0.9700 & $\mathrm{C} 9-\mathrm{O} 2$ & 1.3397 (19) \\
\hline $\mathrm{C} 2-\mathrm{H} 2 \mathrm{~B}$ & 0.9700 & Mo1-O5 & $1.6902(14)$ \\
\hline $\mathrm{C} 3-\mathrm{N} 1$ & $1.275(2)$ & $\mathrm{Mo1}-\mathrm{O} 4$ & $1.7160(13)$ \\
\hline $\mathrm{C} 3-\mathrm{C} 4$ & $1.443(3)$ & Mo1-O1 & $1.9438(12)$ \\
\hline $\mathrm{C} 3-\mathrm{H} 3$ & 0.9300 & $\mathrm{Mo} 1-\mathrm{O} 2$ & $1.9446(12)$ \\
\hline $\mathrm{C} 4-\mathrm{C} 9$ & $1.406(3)$ & Mo1-N1 & $2.2652(14)$ \\
\hline $\mathrm{C} 4-\mathrm{C} 5$ & $1.407(2)$ & $\mathrm{Mo} 1-\mathrm{O} 3$ & $2.3259(14)$ \\
\hline $\mathrm{C} 5-\mathrm{C} 6$ & $1.370(3)$ & $\mathrm{O} 3-\mathrm{H} 1 \mathrm{O}$ & $0.73(2)$ \\
\hline $\mathrm{C} 5-\mathrm{H} 5$ & 0.9300 & $\mathrm{O} 3-\mathrm{H} 2 \mathrm{O}$ & $0.78(3)$ \\
\hline $\mathrm{C} 6-\mathrm{C} 7$ & $1.384(3)$ & & \\
\hline $\mathrm{O} 1-\mathrm{C} 1-\mathrm{C} 2$ & $107.86(16)$ & $\mathrm{C} 7-\mathrm{C} 8-\mathrm{H} 8$ & 119.8 \\
\hline $\mathrm{O} 1-\mathrm{C} 1-\mathrm{H} 1 \mathrm{~A}$ & 110.1 & $\mathrm{C} 9-\mathrm{C} 8-\mathrm{H} 8$ & 119.8 \\
\hline $\mathrm{C} 2-\mathrm{C} 1-\mathrm{H} 1 \mathrm{~A}$ & 110.1 & $\mathrm{O} 2-\mathrm{C} 9-\mathrm{C} 8$ & $117.79(16)$ \\
\hline $\mathrm{O} 1-\mathrm{C} 1-\mathrm{H} 1 \mathrm{~B}$ & 110.1 & $\mathrm{O} 2-\mathrm{C} 9-\mathrm{C} 4$ & $122.62(15)$ \\
\hline $\mathrm{C} 2-\mathrm{C} 1-\mathrm{H} 1 \mathrm{~B}$ & 110.1 & $\mathrm{C} 8-\mathrm{C} 9-\mathrm{C} 4$ & $119.56(16)$ \\
\hline $\mathrm{H} 1 \mathrm{~A}-\mathrm{C} 1-\mathrm{H} 1 \mathrm{~B}$ & 108.4 & $\mathrm{O} 5-\mathrm{Mo1}-\mathrm{O} 4$ & $107.11(7)$ \\
\hline $\mathrm{N} 1-\mathrm{C} 2-\mathrm{C} 1$ & $105.88(15)$ & $\mathrm{O} 5-\mathrm{Mo1}-\mathrm{O} 1$ & $97.32(6)$ \\
\hline $\mathrm{N} 1-\mathrm{C} 2-\mathrm{H} 2 \mathrm{~A}$ & 110.6 & $\mathrm{O} 4-\mathrm{Mo1}-\mathrm{O} 1$ & $96.15(6)$ \\
\hline $\mathrm{C} 1-\mathrm{C} 2-\mathrm{H} 2 \mathrm{~A}$ & 110.6 & $\mathrm{O} 5-\mathrm{Mo1}-\mathrm{O} 2$ & $97.01(6)$ \\
\hline $\mathrm{N} 1-\mathrm{C} 2-\mathrm{H} 2 \mathrm{~B}$ & 110.6 & $\mathrm{O} 4-\mathrm{Mo} 1-\mathrm{O} 2$ & $102.33(6)$ \\
\hline
\end{tabular}




\begin{tabular}{|c|c|c|c|}
\hline $\mathrm{C} 1-\mathrm{C} 2-\mathrm{H} 2 \mathrm{~B}$ & 110.6 & $\mathrm{O} 1-\mathrm{Mo1}-\mathrm{O} 2$ & $152.09(5)$ \\
\hline $\mathrm{H} 2 \mathrm{~A}-\mathrm{C} 2-\mathrm{H} 2 \mathrm{~B}$ & 108.7 & $\mathrm{O} 5-\mathrm{Mo1}-\mathrm{N} 1$ & $90.18(6)$ \\
\hline $\mathrm{N} 1-\mathrm{C} 3-\mathrm{C} 4$ & $124.35(16)$ & $\mathrm{O} 4-\mathrm{Mo} 1-\mathrm{N} 1$ & $161.73(6)$ \\
\hline $\mathrm{N} 1-\mathrm{C} 3-\mathrm{H} 3$ & 117.8 & $\mathrm{O} 1-\mathrm{Mo} 1-\mathrm{N} 1$ & $75.35(5)$ \\
\hline $\mathrm{C} 4-\mathrm{C} 3-\mathrm{H} 3$ & 117.8 & $\mathrm{O} 2-\mathrm{Mo} 1-\mathrm{N} 1$ & $80.78(5)$ \\
\hline $\mathrm{C} 9-\mathrm{C} 4-\mathrm{C} 5$ & $118.37(17)$ & $\mathrm{O} 5-\mathrm{Mo1}-\mathrm{O} 3$ & $166.38(6)$ \\
\hline $\mathrm{C} 9-\mathrm{C} 4-\mathrm{C} 3$ & $122.93(15)$ & $\mathrm{O} 4-\mathrm{Mo} 1-\mathrm{O} 3$ & $86.41(6)$ \\
\hline $\mathrm{C} 5-\mathrm{C} 4-\mathrm{C} 3$ & $118.66(17)$ & $\mathrm{O} 1-\mathrm{Mo1}-\mathrm{O} 3$ & $82.47(5)$ \\
\hline $\mathrm{C} 6-\mathrm{C} 5-\mathrm{C} 4$ & $121.4(2)$ & $\mathrm{O} 2-\mathrm{Mo1}-\mathrm{O} 3$ & $78.05(5)$ \\
\hline $\mathrm{C} 6-\mathrm{C} 5-\mathrm{H} 5$ & 119.3 & $\mathrm{~N} 1-\mathrm{Mo1}-\mathrm{O} 3$ & $76.56(5)$ \\
\hline $\mathrm{C} 4-\mathrm{C} 5-\mathrm{H} 5$ & 119.3 & $\mathrm{C} 3-\mathrm{N} 1-\mathrm{C} 2$ & $121.04(15)$ \\
\hline $\mathrm{C} 5-\mathrm{C} 6-\mathrm{C} 7$ & $119.60(18)$ & $\mathrm{C} 3-\mathrm{N} 1-\mathrm{Mo} 1$ & $127.21(12)$ \\
\hline $\mathrm{C} 5-\mathrm{C} 6-\mathrm{H} 6$ & 120.2 & $\mathrm{C} 2-\mathrm{N} 1-\mathrm{Mo} 1$ & $111.56(11)$ \\
\hline $\mathrm{C} 7-\mathrm{C} 6-\mathrm{H} 6$ & 120.2 & $\mathrm{C} 1-\mathrm{O} 1-\mathrm{Mo} 1$ & $117.80(11)$ \\
\hline $\mathrm{C} 8-\mathrm{C} 7-\mathrm{C} 6$ & $120.62(19)$ & $\mathrm{C} 9-\mathrm{O} 2-\mathrm{Mo} 1$ & $133.92(11)$ \\
\hline $\mathrm{C} 8-\mathrm{C} 7-\mathrm{H} 7$ & 119.7 & $\mathrm{Mo} 1-\mathrm{O} 3-\mathrm{H} 1 \mathrm{O}$ & $114.5(19)$ \\
\hline $\mathrm{C} 6-\mathrm{C} 7-\mathrm{H} 7$ & 119.7 & $\mathrm{Mo} 1-\mathrm{O} 3-\mathrm{H} 2 \mathrm{O}$ & $120.8(18)$ \\
\hline $\mathrm{C} 7-\mathrm{C} 8-\mathrm{C} 9$ & $120.37(19)$ & $\mathrm{H} 1 \mathrm{O}-\mathrm{O} 3-\mathrm{H} 2 \mathrm{O}$ & $109(3)$ \\
\hline $\mathrm{O} 1-\mathrm{C} 1-\mathrm{C} 2-\mathrm{N} 1$ & $-46.2(2)$ & $\mathrm{C} 3-\mathrm{C} 4-\mathrm{C} 9-\mathrm{O} 2$ & $0.6(3)$ \\
\hline $\mathrm{N} 1-\mathrm{C} 3-\mathrm{C} 4-\mathrm{C} 9$ & $8.2(3)$ & $\mathrm{C} 5-\mathrm{C} 4-\mathrm{C} 9-\mathrm{C} 8$ & $0.8(3)$ \\
\hline $\mathrm{N} 1-\mathrm{C} 3-\mathrm{C} 4-\mathrm{C} 5$ & $-174.14(18)$ & $\mathrm{C} 3-\mathrm{C} 4-\mathrm{C} 9-\mathrm{C} 8$ & $178.44(16)$ \\
\hline $\mathrm{C} 9-\mathrm{C} 4-\mathrm{C} 5-\mathrm{C} 6$ & $-1.7(3)$ & $\mathrm{C} 4-\mathrm{C} 3-\mathrm{N} 1-\mathrm{C} 2$ & $-178.44(18)$ \\
\hline $\mathrm{C} 3-\mathrm{C} 4-\mathrm{C} 5-\mathrm{C} 6$ & $-179.50(19)$ & $\mathrm{C} 4-\mathrm{C} 3-\mathrm{N} 1-\mathrm{Mo} 1$ & $7.0(3)$ \\
\hline $\mathrm{C} 4-\mathrm{C} 5-\mathrm{C} 6-\mathrm{C} 7$ & $1.2(3)$ & $\mathrm{C} 1-\mathrm{C} 2-\mathrm{N} 1-\mathrm{C} 3$ & $-149.68(18)$ \\
\hline $\mathrm{C} 5-\mathrm{C} 6-\mathrm{C} 7-\mathrm{C} 8$ & $0.3(3)$ & $\mathrm{C} 1-\mathrm{C} 2-\mathrm{N} 1-\mathrm{Mo} 1$ & $25.66(19)$ \\
\hline $\mathrm{C} 6-\mathrm{C} 7-\mathrm{C} 8-\mathrm{C} 9$ & $-1.3(3)$ & $\mathrm{C} 2-\mathrm{C} 1-\mathrm{O} 1-\mathrm{Mo} 1$ & $51.2(2)$ \\
\hline $\mathrm{C} 7-\mathrm{C} 8-\mathrm{C} 9-\mathrm{O} 2$ & $178.67(17)$ & $\mathrm{C} 8-\mathrm{C} 9-\mathrm{O} 2-\mathrm{Mo} 1$ & $152.01(14)$ \\
\hline $\mathrm{C} 7-\mathrm{C} 8-\mathrm{C} 9-\mathrm{C} 4$ & $0.7(3)$ & $\mathrm{C} 4-\mathrm{C} 9-\mathrm{O} 2-\mathrm{Mo} 1$ & $-30.1(2)$ \\
\hline $\mathrm{C} 5-\mathrm{C} 4-\mathrm{C} 9-\mathrm{O} 2$ & $-177.10(16)$ & & \\
\hline
\end{tabular}

Hydrogen-bond geometry $\left(A,{ }^{\circ}\right)$

\begin{tabular}{lllll}
\hline$D-\mathrm{H} \cdots A$ & $D-\mathrm{H}$ & $\mathrm{H} \cdots A$ & $D \cdots A$ & $D-\mathrm{H} \cdots A$ \\
\hline $\mathrm{O} 3-\mathrm{H} 1 O \cdots \mathrm{O} 1^{\mathrm{i}}$ & $0.73(2)$ & $1.97(3)$ & $2.6656(19)$ & $161(3)$ \\
$\mathrm{O} 3-\mathrm{H} 2 O \cdots 4^{\mathrm{ii}}$ & $0.78(3)$ & $2.07(3)$ & $2.8425(19)$ & $173(3)$ \\
\hline
\end{tabular}

Symmetry codes: (i) $-x+1,-y,-z+1$; (ii) $x,-y+1 / 2, z-1 / 2$. 